\title{
Micromorfologia aplicada a estudos geológico-geotécnicos
}

\author{
Vitor Ribeiro Silos ${ }^{1}$, Helena Polivanov ${ }^{1}$, Leandro Victor dos Santos ${ }^{1}$, Antonio Soares da \\ Silva², Emilio Velloso Barroso', Thiago Teles Álvaro' ${ }^{1}$ \& Bruno Lima de Almeida Cruz ${ }^{1}$
}

\begin{abstract}
Resumo A micromorfologia é uma técnica de estudo de amostras não deformadas de solos e de rochas que permite, com a ajuda de técnicas microscópicas e ultramicroscópicas, identificar os constituintes elementares e as diversas associações destes (esqueleto, plasma, nódulos, poros), além de permitir precisar suas relações mútuas no espaço e, muitas vezes, no tempo. Para o preparo de uma lâmina é necessário a coleta do material em campo, impregnação com resina para seu endurecimento e a posterior laminação. Com as respectivas lâminas prontas realiza-se a descrição micromorfológica de cada amostra coletada. O presente estudo tem como objetivo contribuir, através da análise micromorfológica, no entendimento das características geológicas e geotécnicas de um perfil de alteração de um cambissolo. A análise dos dados micromorfológicos permitiu auxiliar o entendimento do sistema de percolação de água nos diversos horizontes estudados. O horizonte C é o destacadamente mais arenoso entre os materiais analisados e a condutividade hidráulica é semelhante ao dos demais horizontes estudados. Pode-se justificar esse fato pela análise das laminas de micromorfologia, onde se verificou que os poros deste horizonte não são conectados, dificultando a circulação da água livremente. Com isso, pode-se concluir que a micromorfologia pode ser uma ferramenta importante para a compreensão de características geológico-geotécnicas de solos.
\end{abstract}

Palavras-chave: micromorfologia, condutividade hidráulica de solos, solos.

\begin{abstract}
Aplication of micromorphology in geologic-geotechnic studies. The micromorphology is a ultramicroscopic and microscopy technique applicable for grounds and rocks studies in order to identify its primary components and their associations: skeleton, shapes, nodules and hollows. Based on micromorphology it is possible to recognize the mutual relations of those components in space and time. After soil obtaining, a thin section with a resin application is prepared to provoke the hardening allowing the cut. This study has the objective to contribute for the knowledge of the geologic characteristics that can indicate the behavior of an alteration profile of Cambissolo (INCEPTSOL). The analysis of the data allowed to infer about the systems of water percolation in several soil horizons. Horizon $\mathrm{C}$ is more outstandingly rich in sands when compared to the other horizons, but the hydraulic conductivity is very similar to the others horizons. In the images of thin laminas can be verified that the pore presence is not interconnected and, therefore, prevent the free circulation of water. It was possible to conclude that the micromorphology is an important tool for understanding the geologic-geotechnics characteristics and help in the interpretation of the geotecnic behavior.
\end{abstract}

Keywords: micromorphology, hydraulic conductivity, soil.

INTRODUÇÃO A micromorfologia vem sendo documentada desde a década de quarenta do século passado (Kubiena 1938). É uma ferramenta importante que permite observar os componentes estruturais do solo na sua forma natural, possibilitando melhor visualização do comportamento da estrutura e do espaço poroso para a compreensão de muitos problemas identificados no campo, em diversas áreas da ciência do solo (Brewer 1976, Leprun 1979, Bullock et al. 1985).

Essa técnica é uma complementação às análises realizadas convencionalmente, em questões associadas à gênese e manejo dos solos, a biologia do solo, a geotecnia e a geologia de engenharia e ambiental entre outras. Sua utilização auxilia na compreensão da mineralogia, química e física dos solos; análises das suas transformações estruturais; estudos da atividade biológica no solo; qualificação e quantificação do índice de vazios; caracterização dos agregados e indicação do seu comportamento mecânico; estimativa granulométrica; caracterização dos tipos de ligações estruturais; determinação do grau de isotropia e homogeneidade entre outras.

Trabalhos pioneiros, usando análise de imagens para quantificar e caracterizar a porosidade de amostras de solos impregnadas, foram realizados por Jongerius et al. (1972) e Murphy et al. (1977 a, b). Bouma et al. (1977) introduziram o uso desta técnica no estudo da continuidade dos poros. Bullock \& Thomasson (1979) fizeram comparações de medidas de macroporosidade, derivadas da análise de imagens, com aquelas oriundas

1 - UFRJ - Universidade Federal do Rio de Janeiro, Rio de Janeiro (RJ), Brasil. E-mail: vitorsilos@gmail.com, hpolivanov@gmail.com, leovictor22@yahoo.com.br, emilio@geologia.ufrj.br, thteal@gmail.com,brunolacruz@gmail.com 2 - UERJ - Universidade do Estado do Rio de Janeiro, Rio de Janeiro (RJ), Brasil. E-mail: antoniossoares@gmail.com 
da retenção de água, método comumente utilizado na determinação da distribuição do tamanho de poros. Yurong et al. (2006) fizeram o uso da micromorfologia para ajudar no controle de escorregamentos de solos e Wongpokhoma et al. (2008) utilizaram o estudo micromorfológico para quantificar o efeito da salinidade nos solos da Tailândia.

No Brasil, a micromorfologia teve início na década de 70 com trabalhos que tinham como objetivo descrever características micromorfológicas, tais como cutans (Falci \& Mendes 1973), técnicas de impregnação de amostras de solo (Mendes et al. 1973); gênese de pequenos agregados arredondados (Moura Filho \& Buol 1976); e como técnica auxiliar para interpretações pedogenéticas (Dematté et al. 1977). Atualmente a micromorfologia vem sendo usada para trabalhos aplicados, podendo-se citar Juhász et al. (2007), os quais estudaram o uso dos atributos da micromorfologia, como a porosidade, relacionados com a retenção de água no solo. Oliveira (2006) buscou, através da micromorfologia, estudar a evolução do grau de intemperismo de um perfil de solo.

O fator motivador para este estudo é a aplicação da micromorfologia para a compreensão do comportamento geotécnico de solos residuais e coluviais, levando em consideração, principalmente, o que concerne aos padrões estruturais dos materiais analisados. Os parâmetros estruturais influenciam diretamente no desempenho de solos em obras civis e na percolação de fluidos e transporte de contaminantes através dos mesmos. Outro fator é a possibilidade da definição das características microestruturais que possam servir como indicadoras da evolução do grau de intemperismo de perfis de solos, associando essas características com o comportamento hidráulico dos solos.

A área de estudo está compreendida dentro da Faixa Ribeira (Almeida et al. 1973), mais precisamente no Arco Magmático Rio Negro (Tupinambá 1999). As unidades inseridas dentro deste contexto são: Complexo Rio Negro, Batólito Serra dos Órgãos, Granito Teresópolis, Diques e Coberturas Aluvionares. A unidade mais representativa da área é o Batólito Serra dos Órgãos, que ocupa cerca de $60 \%$, quase o dobro da área coberta pelo complexo Rio Negro que ocupa $33 \%$, enquanto que as intrusões pós-tectônicas da unidade Granito Teresópolis ocupam 6\%. O 1\% restante são os diques e as coberturas quaternárias (Santos 2008).

Geomorfologicamente, Dantas et al. (2000) inseriram a área na unidade Geomorfológica Escarpas das Serras do Couto e dos Órgãos. Esta unidade consiste em uma muralha montanhosa, alçada por tectônica, que delimita o recôncavo da Baía de Guanabara. Esta unidade caracteriza-se como uma barreira orográfica abrupta. É comum a ocorrência de movimentos de massa nas escarpas florestadas, demonstrando o processo de evolução geomorfológica dessa unidade de relevo.

Os solos predominantes regionalmente são LATOSSOLOS (OXISOL) e CAMBISSOLOS (INCEPT$\mathrm{SOL}$ ). Os primeiros são desenvolvidos devido ao intenso intemperismo propiciado pelo grande volume de chuvas que caem na área. Os CAMBISSOLOS estão associados aos locais mais íngremes, que promovem intensa remoção dos materiais intemperizados (Carvalho Filho et al. 2000).

OBJETIVOS O presente trabalho tem como objetivo estudar as características micromorfológicas de um perfil de CAMBISSOLO (INCEPTSOL) buscando compreender a sua evolução intempérica, através da identificação dos constituintes presentes na fração sólida e os arranjos ou modos de organização do esqueleto e plasma com os poros, correlacionando-os com a condutividade hidráulica desses materiais.

MATERIAIS E MÉTODOS Para o presente estudo foi selecionado um perfil de CAMBISSOLO, situado próximo à rodovia BR-116, no bairro Jardim Meudon, no município de Teresópolis - RJ (Fig. 1), situado na região Sudeste do Brasil.

A região escolhida para este estudo apresenta um perfil de solo desenvolvido in situ com uma cobertura coluvionar. Neste perfil observou-se uma sequência de horizontes A-B-C (Fig. 2) com horizonte B pedologicamente pouco evoluído, marcado pela presença de minerais herdados do material original, pouco intemperizado. O horizonte A possui pequena espessura, em torno de $40 \mathrm{~cm}$, e é caracterizado por uma coloração marrom escura devido aos materiais orgânicos presentes. O horizonte B apresenta uma espessura aproximada de 150 $\mathrm{cm}$, sendo definido como horizonte B incipiente, de coloração amarelada, textura média, cerosidade pouco expressiva, com desenvolvimento de estrutura em blocos muito fraca, fragmentos e seixos provenientes da rocha original. O horizonte $\mathrm{C}$ guarda as características da decomposição mineral e textural da rocha-matriz. Apresenta coloração avermelhada e textura arenosa (Fig. 3).

Para a realização da análise micromorfológica foram executadas as seguintes etapas: amostragem de solo com estrutura indeformada (micromonólitos), impregnação, laminação, polimento e interpretação no microscópio (Fig. 4).

As amostras indeformadas, ou seja, extraídas com o mínimo de perturbação, mantendo sua estrutura e condições de umidade e compacidade preservadas, foram coletadas conforme a norma NBR-9604 e acomodadas em pequenas caixas de papelão com dimensões de $7 \mathrm{~cm} \mathrm{x} 5 \mathrm{~cm} \mathrm{e} 4 \mathrm{~cm}$ de altura.

Estas amostras foram orientadas, possibilitando a identificação do topo do perfil, viabilizando o estudo da direção do transporte de material dentro do solo. Posteriormente, as mesmas foram secas ao ar livre para eliminação do excesso de umidade. Após esses procedimentos, foi realizada a impregnação dos blocos das amostras indeformadas com o objetivo de torná-las resistentes para que fossem laminadas. O material foi impregnado com uma mistura de resina plástica Araldite XGY-1109 100\%, 10\% de endurecedor HY-951 e 40 a $50 \%$ de acetona P.A. As condições de impregnação não devem afetar o arranjo dos constituintes ou mudar-lhes 


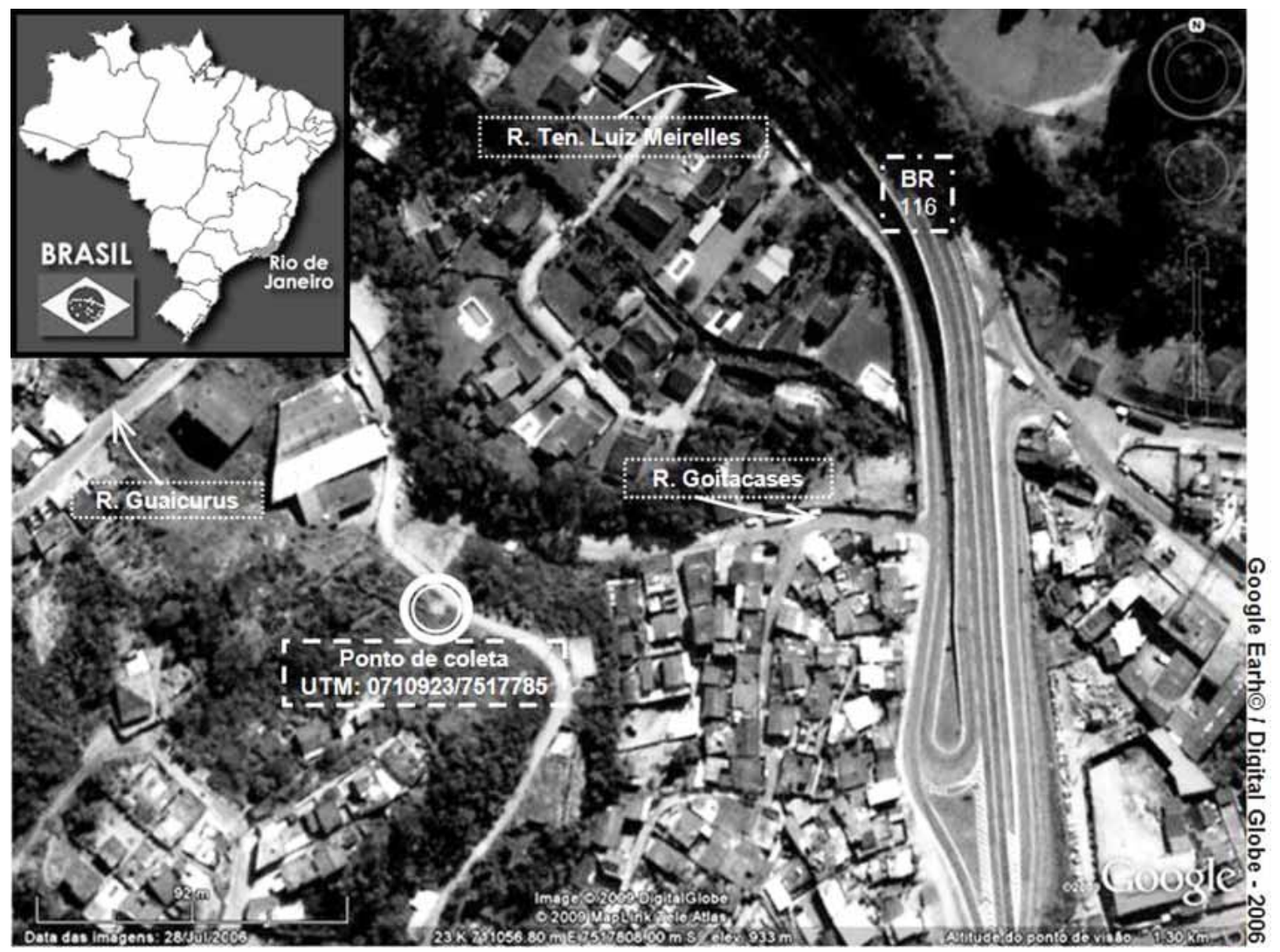

Figura 1 - Localização - imagem retirada do Google Maps (2006).

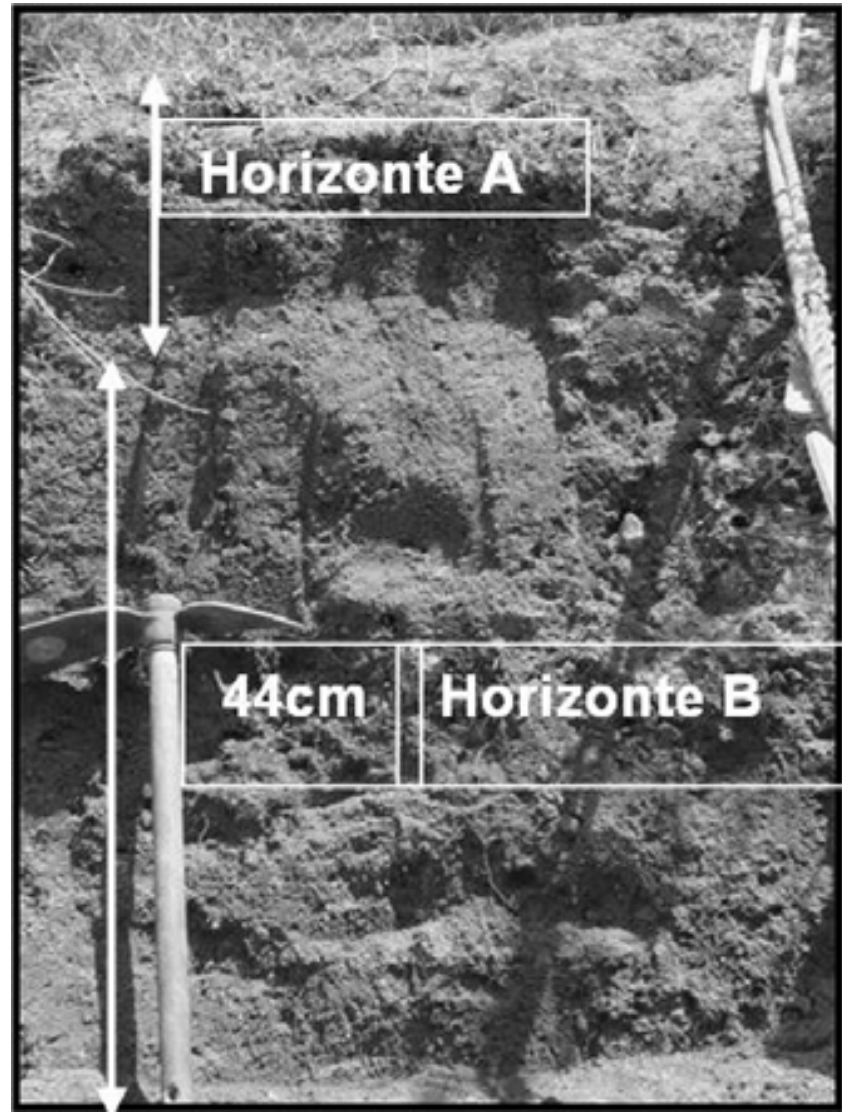

Figura 2 - Horizonte A sobrejacente ao B, diagnóstico do Cambissolo.

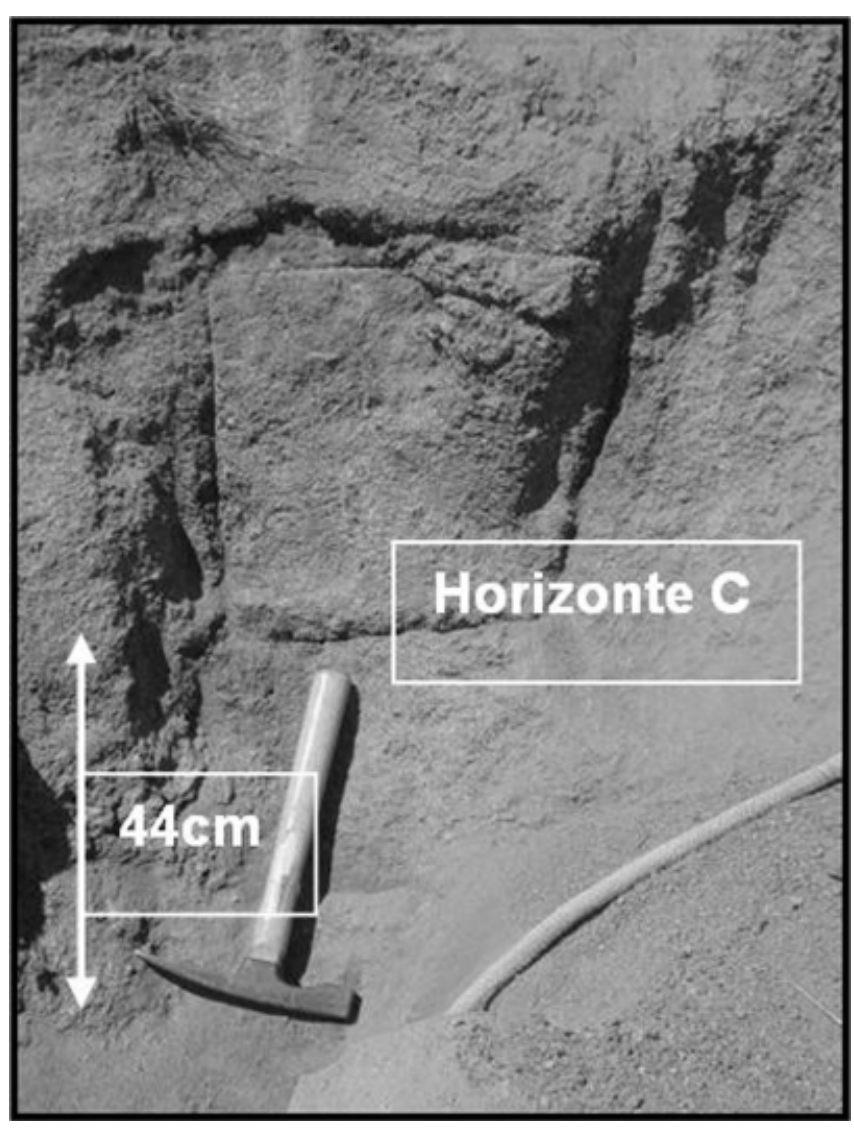

Figura 3 - Horizonte C com evidentes características da rocha de origem. 


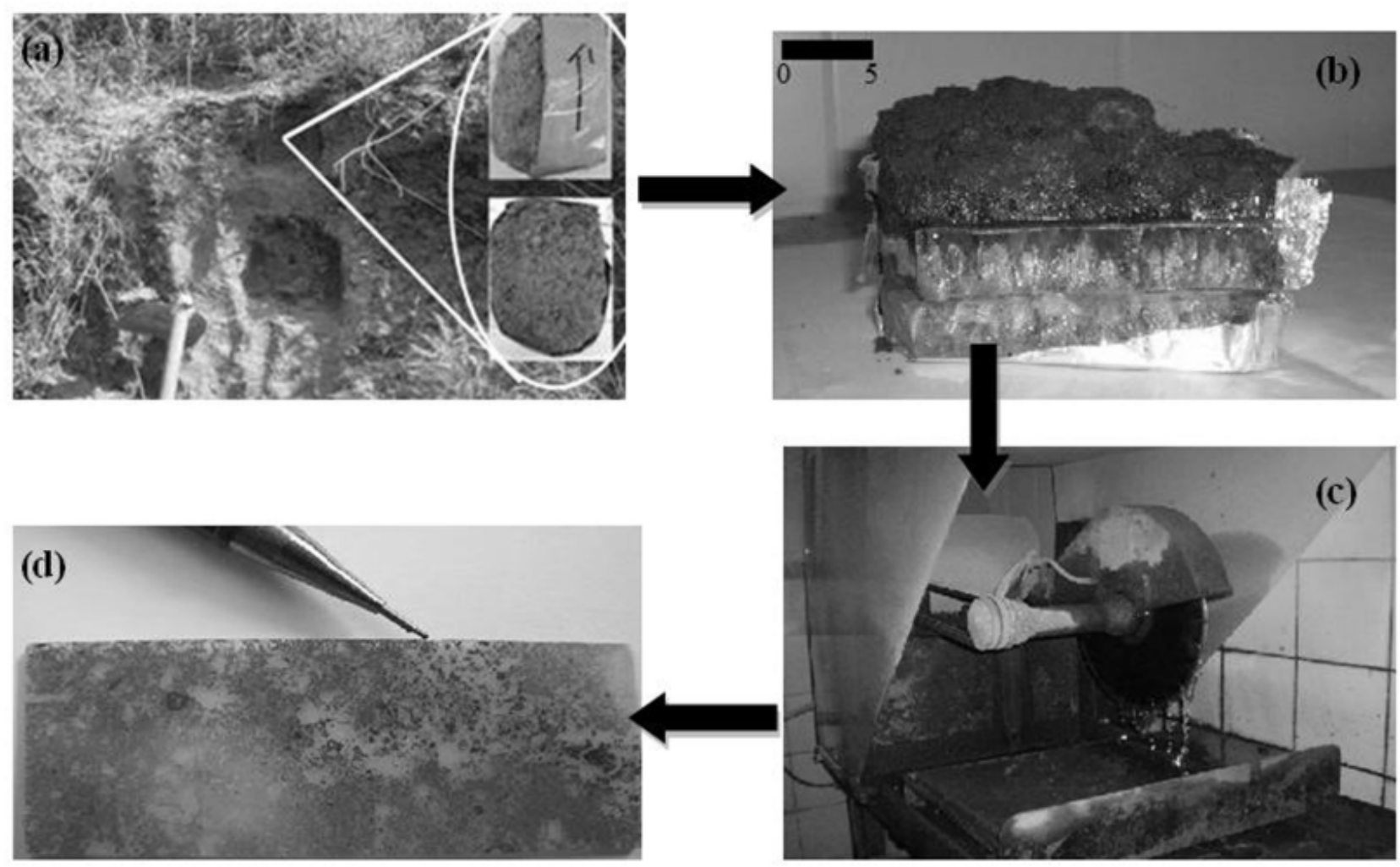

Figura 4 - Fluxograma contendo as etapas para a confecção de lâminas das amostras de solo. (a) coleta das amostras em perfil de solo; (b) amostra de solo impregnada com resina; (c) serra para laminação; (d) lâmina de solo.

as propriedades ópticas. Desta forma, optou-se por uma resina plástica conhecida comercialmente como Araldite, que apresenta baixa toxicidade, endurecimento e polimerização mais rápidos e grau de contração mais baixo do que a da resina de poliéster.

Para cada amostra, foram realizadas pelo menos três sessões de impregnação por capilaridade obtida à vácuo seco, em intervalos de $24 \mathrm{~h}$, para melhor penetração da resina na amostra. Neste processo foi utilizada uma bomba de vácuo conectada a um dessecador que abriga a resina e as amostras. Com a polimerização, a amostra tornou-se rija, resistente, mas sem perder a estrutura original, o que permitiu proceder com o seu corte e a sua laminação. $O$ endurecimento completo das amostras ocorreu após duas a três semanas. Houve essa diferença de tempo devido às diferentes características dos materiais.

Depois do endurecimento das amostras deu-se sequência à sua laminação. Cada bloco foi cortado em máquina de disco diamantado em duas partes e, por último, cada fatia foi polida em um disco em rotação com abrasivo e água (Fig. 5), até alcançar uma superfície lisa e plana o suficiente para ser colada em lâmina de vidro. As amostras coladas foram desbastadas e polidas manualmente com abrasivo (carborundum) até a espessura de 30 micra quando, finalmente, o material ficou adequado para estudos microscópicos.

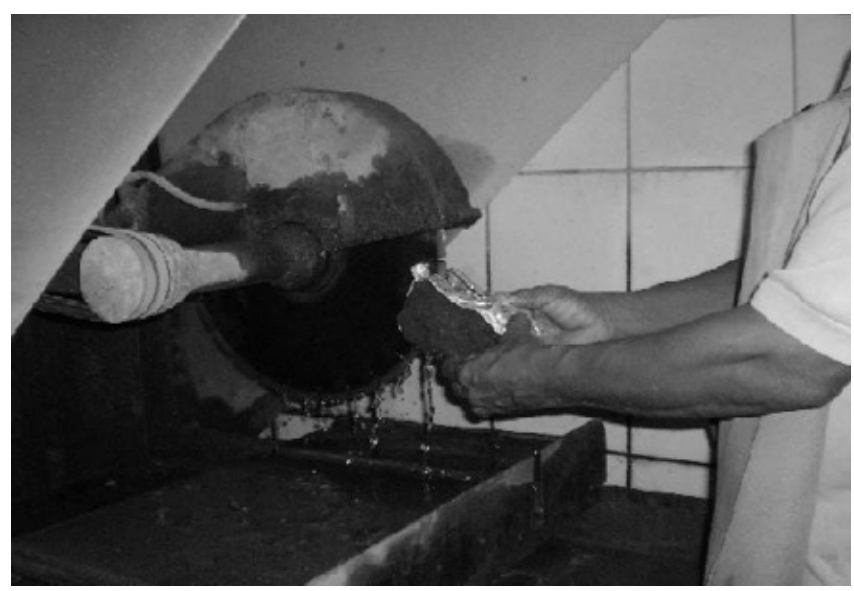

Figura 5 - Fase de corte, do solo impregnado por resina, para laminação.

A caracterização granulométrica foi realizada empregando-se os métodos do peneiramento e da sedimentação conjugados, segundo as recomendações prescritas na norma NBR 7181 (ABNT 1984).

Nos ensaios de laboratório para a determinação da condutividade hidráulica dos horizontes do solo estudado, os corpos de prova foram obtidos por puncionamento de um cilindro de acrílico com 4,9 cm de 
diâmetro no bloco de solo coletado no campo. O tubo de acrílico foi levado ao permeâmetro de carga constante e foram seguidos os procedimentos de ensaio da NBR 13292 (ABNT 1995).

RESULTADOS OBTIDOS Os resultados dos ensaios de permeabilidade e análises granulométricas foram obtidos em (Santos 2008). Os percentuais granulométricos para os horizontes A, B e C estão demonstrados na tabela 1 .

Os valores da condutividade hidráulica, determinados nos horizontes $\mathrm{A}, \mathrm{B}$ e C, estão demonstrados na tabela 2.

Em cada horizonte foram realizadas as análises micromorfológicas e estas correlacionadas com os resultados granulométricos e da condutividade hidráulica, buscando compreender as relações entre essas características dos solos. As lâminas foram elaboradas para acrescentar informações sobre o solo estudado, permitindo o melhor entendimento da distribuição de seus microporos, microestruturas e microtexturas.

As observações de campo permitiram caracterizar o horizonte A como um colúvio (Fig. 6), os resultados da micromorfologia mostraram que este é um horizonte pouco retrabalhado devido ao pequeno transporte a que foi submetido. As evidências deste fato estão calcadas na observação das lâminas, onde foi constatada a presença de grãos muito angulosos e de feldspatos alterados (pseudomorfisados) ao lado de feldspatos não alterados. A porosidade encontrada é caracteristicamente cavitária e de empilhamento bem conectada, os agregados são ligeiramente arredondados e de tamanho pequeno. Logo, pode se associar a estas características micromorfológicas uma maior facilidade à circulação de fluídos devido à alta conectividade dos poros.

O horizonte B (Fig. 7), apesar de maduro, ainda preserva características do material de origem, com minerais pouco intemperizados, e não foram observadas

Tabela 1 - Porcentuais granulométricos.

\begin{tabular}{lll}
\hline & $\begin{array}{l}\text { Profundidade } \\
(\mathrm{cm})\end{array}$ & $\begin{array}{l}\text { Condutividade hidráulica } \\
(\mathrm{cm} / \mathrm{s})\end{array}$ \\
\hline Horizonte A & 40 & $4,7 \times 10^{-3} \mathrm{~cm} / \mathrm{s}$ \\
\hline Horizonte B & 190 & $5,4 \times 10^{-3} \mathrm{~cm} / \mathrm{s}$ \\
\hline Horizonte C & 490 & $1,8 \times 10^{-3} \mathrm{~cm} / \mathrm{s}$ \\
\hline
\end{tabular}

Tabela 2 - Condutividade hidráulica.

\begin{tabular}{lcccc}
\hline & Pedregulho & Areia & Silte & Argila \\
\hline Horizonte A & $9 \%$ & $52 \%$ & $14 \%$ & $25 \%$ \\
\hline Horizonte B & $12 \%$ & $54 \%$ & $15 \%$ & $18 \%$ \\
\hline Horizonte C & $4 \%$ & $78 \%$ & $16 \%$ & $2 \%$ \\
\hline
\end{tabular}

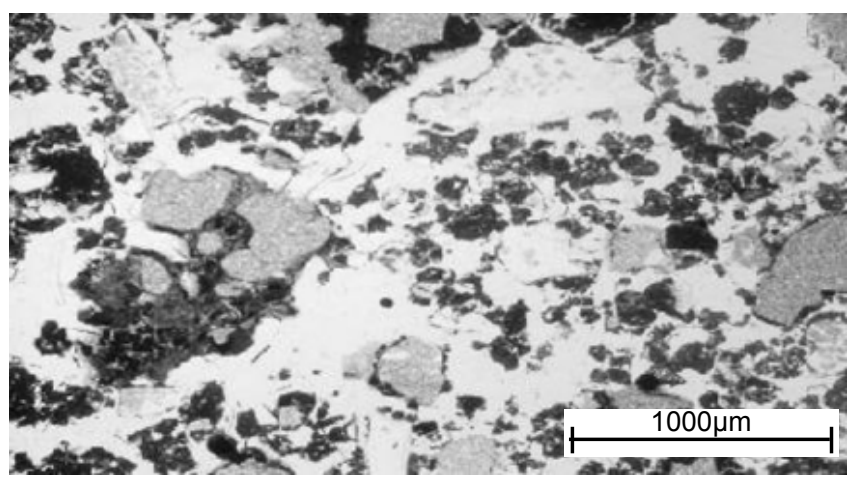

Figura 6 - Lâmina delgada do horizonte A.

descontinuidades entre este horizonte e o localizado imediatamente abaixo. O horizonte $\mathrm{B}$ apresenta poros fissurais e cavitários assim como agregados de maior tamanho que aqueles presentes no horizonte A. No horizonte $\mathrm{B}$, a presença de poros cavitários e fissurais oferece uma maior resistência à circulação de fluídos. O plasma (fração argila) aparece em maior quantidade quando comparado com o horizonte $\mathrm{C}$.

O horizonte C (Fig. 8) é um saprólito e preserva as características da rocha de origem. A descrição das lâminas permitiu classificar a porosidade como cavitária, que neste caso é resultante da dissolução de minerais, sendo essa pouco ou não conectada, promovendo um forte impedimento à circulação de fluidos. Este horizonte marca o início da formação do plasma (alteroplasmação). Este plasma ainda não é suficiente para criar microestruturas pedogenéticas. No esqueleto as frações predominantes são areia e silte.

CONCLUSÕES Foi possível concluir que o horizonte A é transportado e que os horizontes B e C são formados in situ a partir da evolução da alteração da rocha.

As porosidades dos horizontes pedológicos $\mathrm{A}$ e do B são mais funcionais do que a do horizonte C. No horizonte $\mathrm{B}$ a pedogênese possibilitou a formação de estrutura em blocos, à semelhança do que foi verificado no campo, e poros fissurais. Certas feições como,

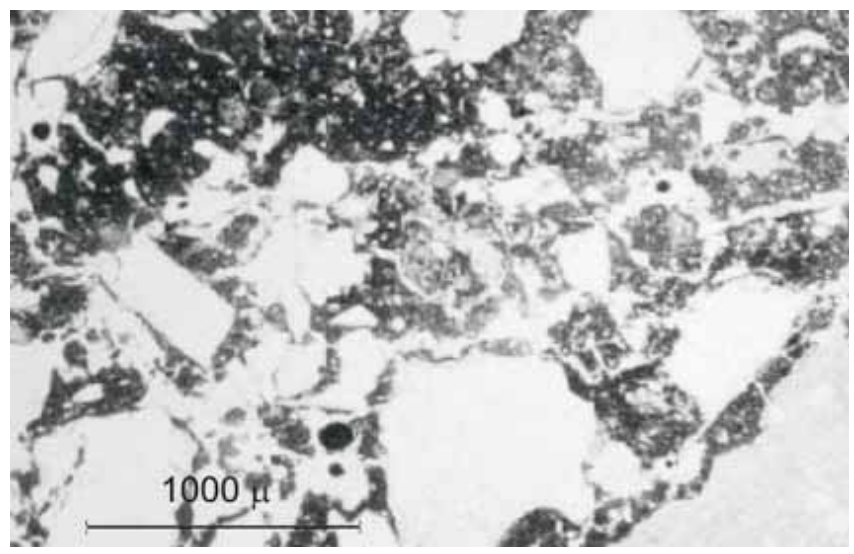

Figura 7 - Lâmina delgada do horizonte B. 


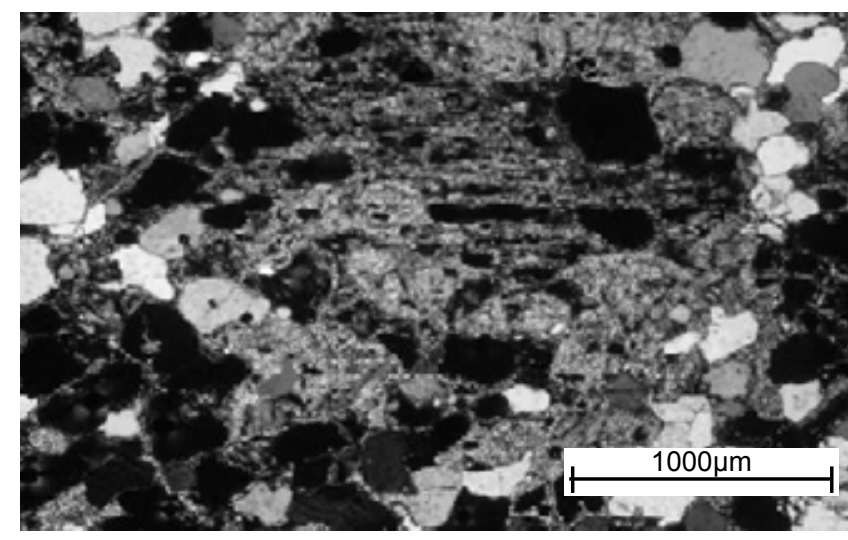

Figura 8 - Lâmina delgada do horizonte $C$.

por exemplo, os box work de gibbsita, no horizonte A, quando associadas às características de campo e morfologia dos minerais (subangulares) são indicadores de que este horizonte é coluvial com transporte de curta distância. No saprólito (horizonte C) a porosidade de dissolução de minerais é pouco conectada sendo um impedimento à circulação de fluídos. Este horizonte apesar de arenoso, com elevados teores de silte e com grande volume de poros, possui condutividade hidráulica semelhante aos horizontes A e B, que são bem mais argilosos. A micromorfologia possibilitou demonstrar que um horizonte de textura arenosa e elevado volume de poros possui condutividade hidráulica da mesma ordem de grandeza de solos mais finos, devido a pouca conectividade existente entre os seus poros.

A identificação de solos maduros, quanto a sua origem (residual ou transportado), é um aspecto que pode ser de difícil identificação no campo e é um problema recorrente na Geologia de Engenharia, pois é distinto o comportamento geotécnico de solos transportados e residuais. Dessa forma, a micromorfologia associada ao trabalho de campo demonstra ser uma ferramenta importante para essa distinção. A descrição micromorfológica foi importante na compreensão do sistema de percolação de fluidos nos horizontes estudados.

\section{Referências}

ABNT - ASSOCIAÇÃO BRASILEIRA DE NORMAS TÉCNICAS. 1984. NBR 7181: Solo - Análise Granulométrica (método de ensaio). Rio de Janeiro, 1984.

ABNT - ASSOCIAÇÃO BRASILEIRA DE NORMAS TÉCNICAS. 1986. NBR 9604: Solo - Abertura de poço e trincheira de inspeção em solo, com retirada de amostras deformadas e indeformadas. Rio de Janeiro, 1986.

Almeida F.F.M., Amaral G., Cordani U.G., Kawashita K. 1973. The Precambrian evolution of the South American cratonic margin south of Amazon River. In: Nairn E.M. \& Stheli F. (eds.) The ocean basins and margins. New York, Plenum, p. 411-446.

Bouma J., Jongerius A., Boersma O.H., Jager,A, Schoonderbeek D. 1977. The function of different types of macropores during saturated flow through four swelling soil horizons. Soil Science Society of America Journal, 41:945-950.

Brewer R. 1976. Fabric and mineral analysis of soils. New York, R.E. Krieger, 73 p.

Bullock P., Thomasson A.J. 1979. Rothamsted studies of soil structure. II. Measurement and characterization of macroporosity by image analysis and comparison with data from water retention measurements. Journal of Soil Science, 30:391-413.

Bullock P., Fedoroff N., Jongerius A., Stoops G., Tursina T. 1985. Handbook for soil thin section description. Wolverhampton, Waine Research Publisher, $152 \mathrm{p}$.

Carvalho Filho A., Lumbreiras J.F., Santos R.D. 2000. Os Solos do Estado do Rio de Janeiro. Brasília, CPRM, 1 CD-Rom.

Dantas M.E., Shinzato E., Medina A.I.M., Silva C.R., Pimentel J., Lumbreiras J.F., Calderano S.B., Cavalho Filho A. 2000. Dignóstico Geoambiental do Estado do Rio de Janeiro. Brasília, CPRM, 1 CD-Rom.

Dematté J.L.I., Wilding L.P., Holowaychuk N. 1977. Solos da região de São Pedro, Estado de São Paulo. III. Micromorfologia. Revista Brasileira de Ciência do Solo, 1:104-107.

Falci S.C., Mendes A.C.T. 1973. Identificação de cutans em perfis de latossolo roxo e terra roxa estruturada. Anais da ESALQ, 30:49-70.

GOOGLE MAPS 2006. Disponível em: http://whttp://maps. google.com.br/. Acessado em: 15/09/2009.

Jongerius A., Schooderbeek D., Jager A. 1972. The application of the Quantimet 720 in soil micromorphometry. The Microscope, 20:243-254.

Juhász C.E.P., Cooper M., Cursi P.R., Ketzer A.O., Toma R.S. 2007. Micromorfologia de solo sob cerradão em relação à retenção de água. Sci. Agric., 64(4):344-354

Kubiena W.L. 1938. Micropedology. Ames, Collegiate Press, $243 \mathrm{p}$.

Leprun J.C. 1979. Les cuissares ferrugiuneses des pays cristallins de l'Afrique Occidentale sèche - Gènese, transformation, dégradation. Tese de Doutorado, Université Louis Pasteur de Strasbourg, Strasbourg, $208 \mathrm{p}$.

Mendes A.C.T., Falci S.C., Dematté J.L.I. 1973. Secções delgadas de solos: método de impregnação. Anais da ESALQ, 30:35-48.

Moura Filho W., Buol S.W. 1976. Studies of a Latosol Roxo (Eutrustox) in Brazil: Micromorphology effect on ion release. Experientiae, 21:161-177.

Murphy C.P., Bullock P., Turner R.H. 1977a. The measurement and characterization of voids in soil thin sections by image analysis: Part I: Principles and techniques. Journal of Soil Science, 28:498-508.

Murphy C.P., Bullock P., Turner R.H. 1977b. The measurement and characterization of voids in soil thin sections by image analysis: Part I: Applications. Journal of Soil Science, 28:509-518. 
Oliveira E.P. 2006. Caracterização bio-físico-químicomineralógica e micromorfológica de um perfil de alteração de granito-gnaisse de Curitiba, PR. Dissertação de mestrado, Departamento de Engenharia Civil, Pontifícia Universidade Católica do Rio de Janeiro, Rio de Janeiro, 197 p.

Santos L.V. 2008. Estudo da influência da bentonita em um solo residual para uso como camada selante. Dissertação de Mestrado, COPPE - Instituto Alberto Luiz Coimbra de Pós-Graduação e Pesquisa de Engenharia, Universidade Federal do Rio de Janeiro, Rio de Janeiro, 139 p.

Tupinambá M. 1999. Evolução tectônica e magmática da Faixa Ribeira na região serrana do Estado do Rio de
Janeiro. Tese de Doutorado, Instituto de Geociências, Universidade de São Paulo, São Paulo, 221 p.

Yurong H., Peng C., Chaolin L., Baohua Z., YU Z. 2006 Micromorphology of landslide soil. Journal of Mountain Science, 3:36-50.

Wongpokhoma N., Kheoruenromnea I., Suddhiprakarna A., Gilkesb R.J. 2008. Micromorphological properties of salt affected soils in Northeast Thailand. Geoderma, 144:158-170.

Manuscrito ID 16501

Submetido em 30 de dezembro de 2009 Aceito em 01 de dezembro de 2010 